\title{
Novel Composite Based on Bimetallic AuNi-Embedded Nano X Zeolite/MWCNT as a Superior Electrocatalyst for Oxygen Evolution Reaction
}

\author{
Fatemeh Amiripour $^{\mathrm{a}}$, Shahram Ghasemi ${ }^{\mathrm{b}, *}$, Seyed Naser Azizi ${ }^{\text {a }}$
}

${ }^{a}$ Analytical Division, Faculty of Chemistry, University of Mazandaran, Beheshti Street, 47416-95447, Babolsar, Iran

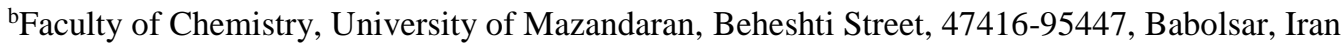

*Corresponding to Shahram Ghasemi, e-mail: sghasemimir@yahoo.com

, sghasemi@umz.ac.ir, Tel/fax: +981135302388.

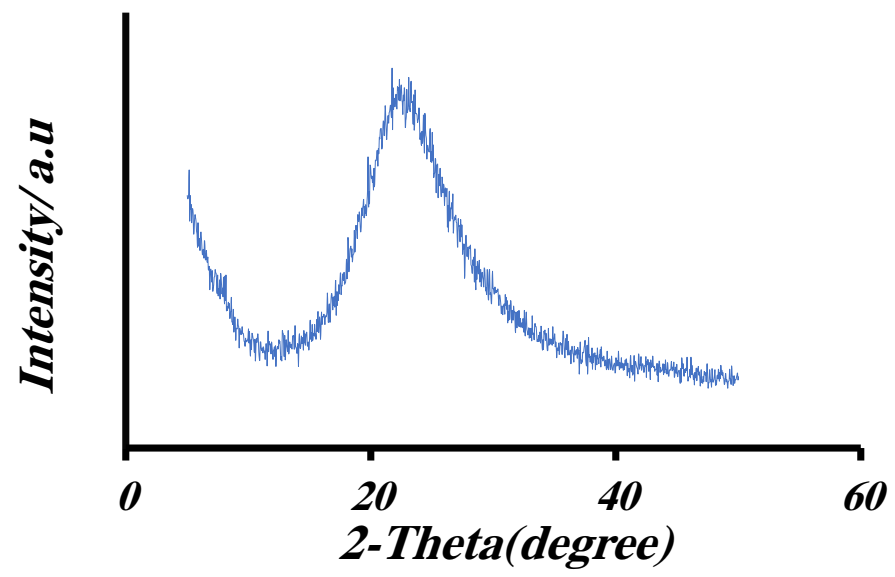

Fig. S1. XRD pattern of SSA.

Table S1. wt. \% of elements obtained from EDS analysis for different samples.

\begin{tabular}{|c|c|c|c|c|c|c|}
\hline Sample & $\mathbf{O}$ & $\mathbf{N a}$ & Al & $\mathbf{S i}$ & $\mathbf{N i}$ & Au \\
\hline NXZ & 52.02 & 6.57 & 17.38 & 24.02 & - & - \\
\hline NiNXZ & 47.64 & 6.62 & 14.58 & 21.55 & 9.60 & - \\
\hline AuNiNXZ & 45.65 & 4.15 & 12.49 & 16.88 & 6.21 & 14.61 \\
\hline
\end{tabular}



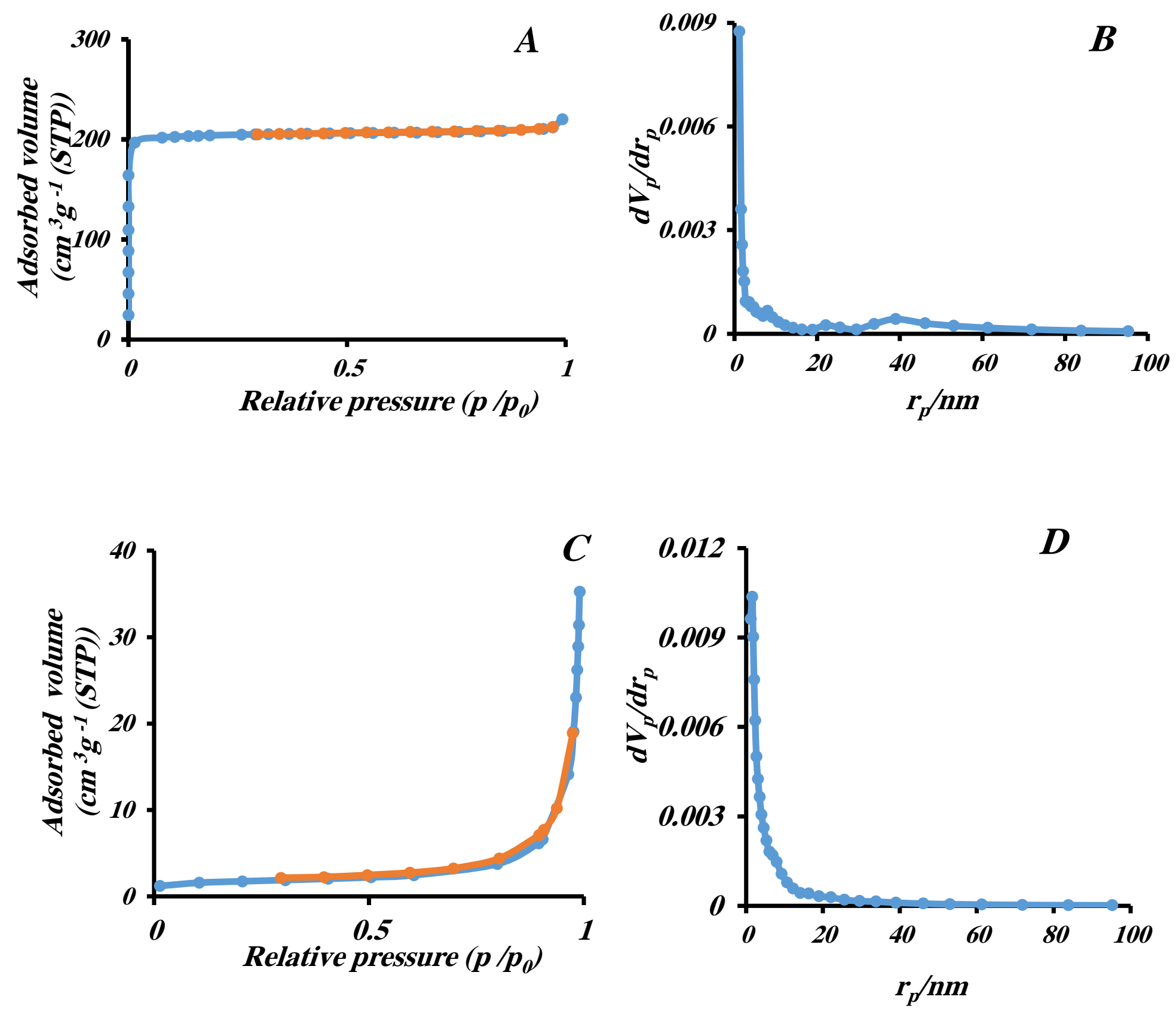

Fig. S2. Nitrogen adsorption-desorption isotherms and pore size distribution of NXZ (A, B) and AuNiNXZ (C, D). 

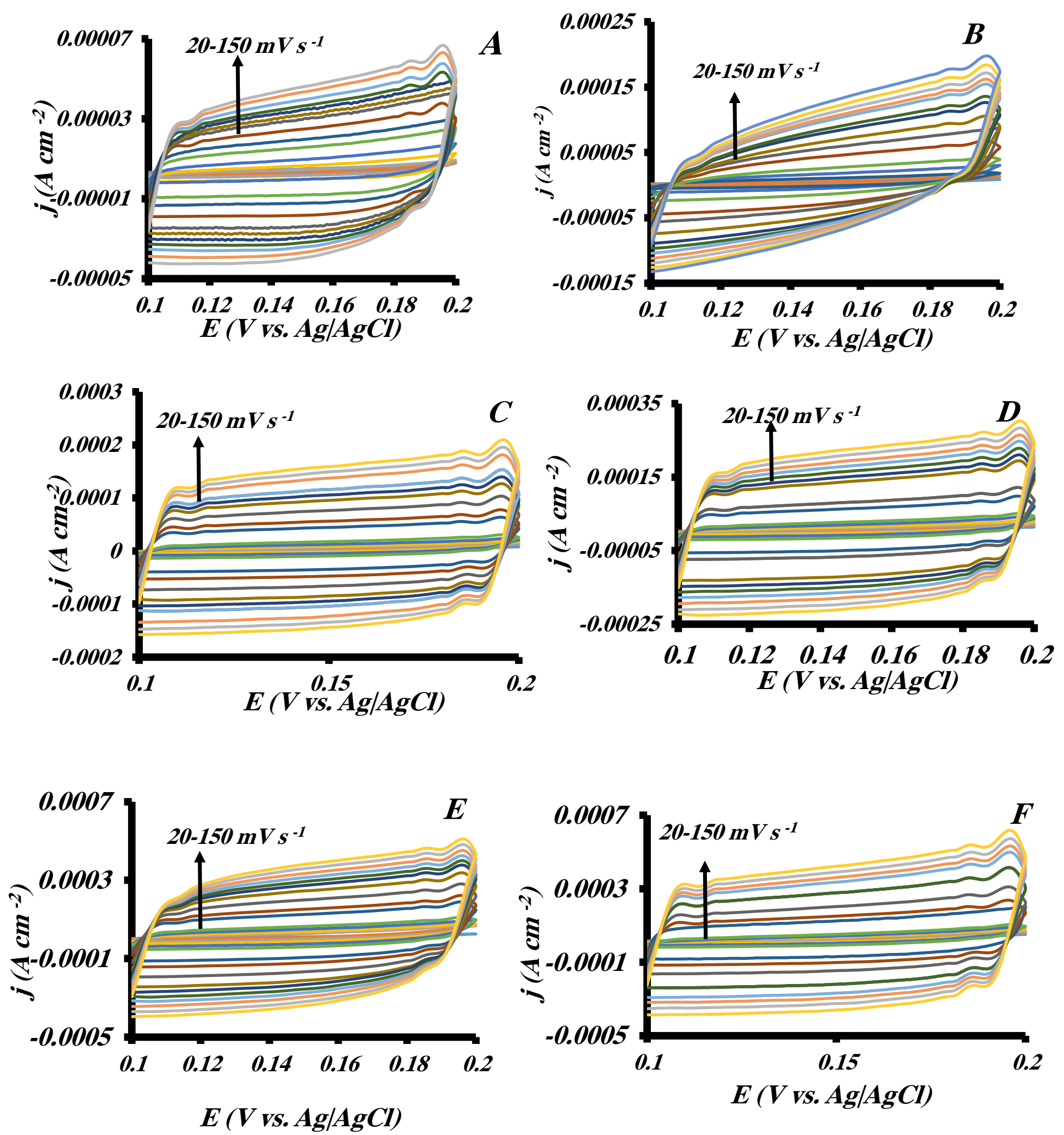

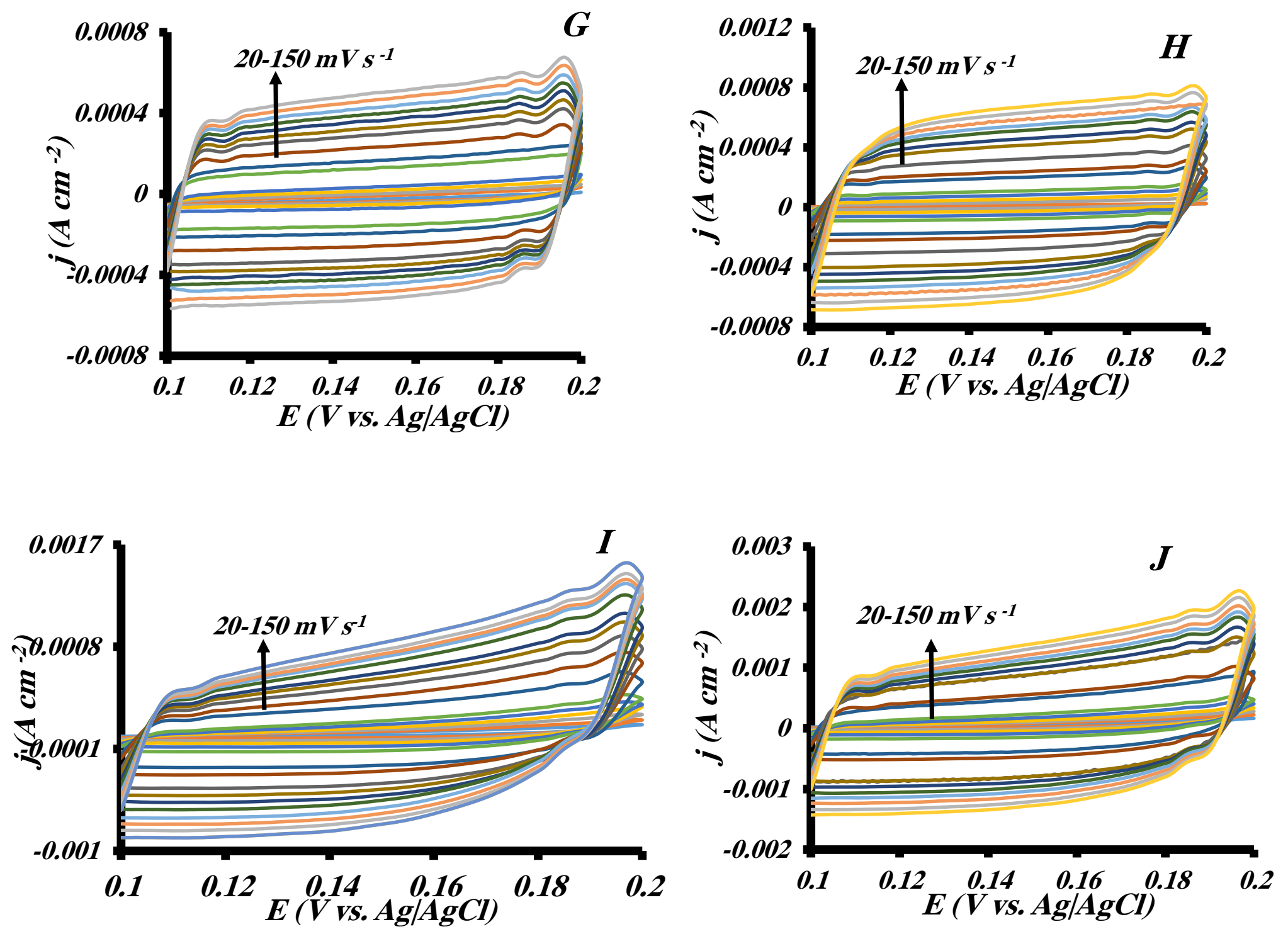

Fig. S3. Cyclic voltammograms in the double layer region of the CPE (A), NXZ/CPE (B), MWCNT/CPE (C),

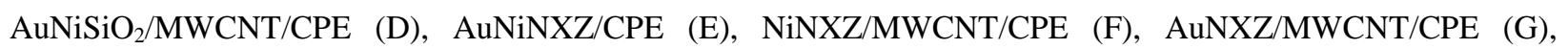
AuNiNXZ $10 / \mathrm{MWCNT}_{25} / \mathrm{CPE}_{65}(\mathrm{H}), \mathrm{AuNiNXZ}_{25} / \mathrm{MWCNT}_{10} / \mathrm{CPE}_{65}$ (I) and $\mathrm{AuNiNXZ}_{25} / \mathrm{MWCNTs}_{25} / \mathrm{CPE}_{50}(\mathrm{~J})$ at scan rates ranging from 20 to $150 \mathrm{mV} \mathrm{s}^{-1}$. 


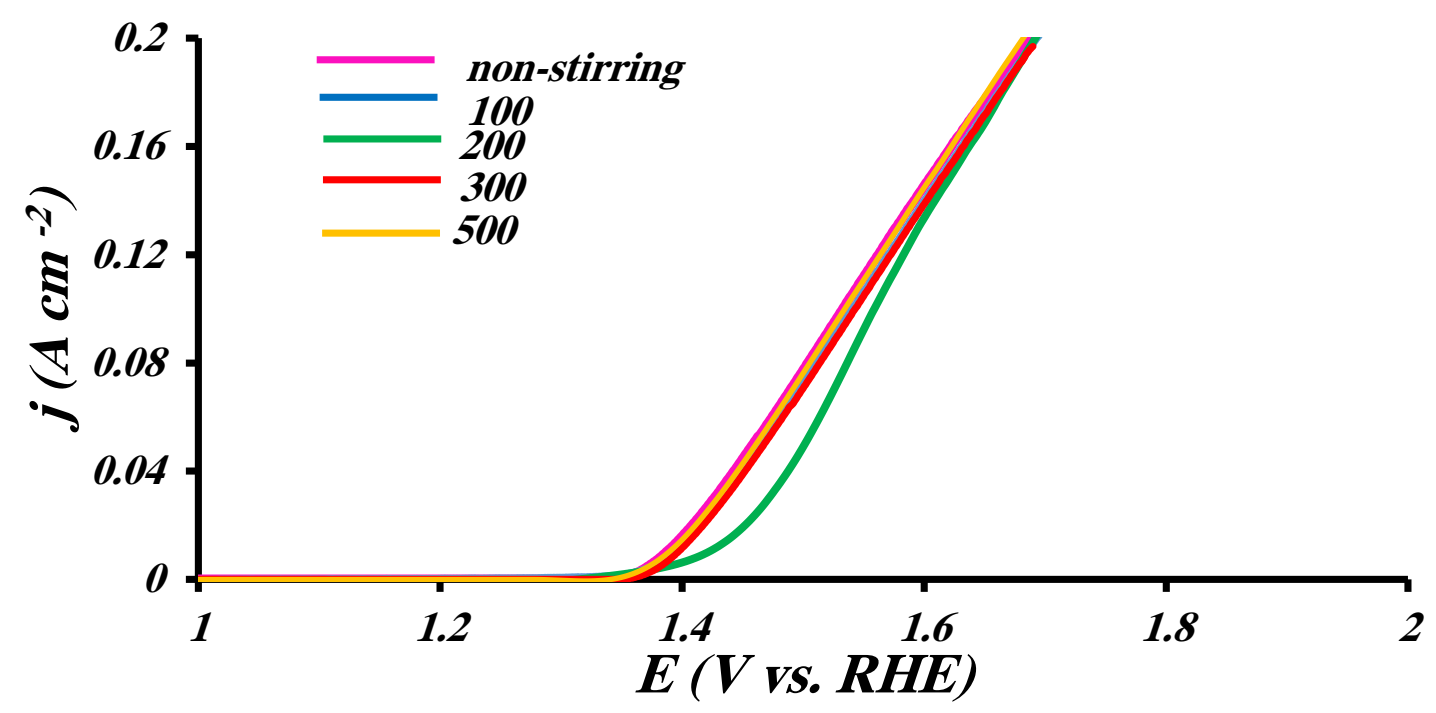

Fig. S4. Linear sweep voltammetry of $\mathrm{AuNiNXZ}_{25} / \mathrm{MWCNT}_{25} / \mathrm{CPE}_{50}$ under non-stirring and stirring at different rpms.

Table S2. Values of the equivalent circuit parameters obtained by fitting the experimental data of Nyquist plots.

\begin{tabular}{|c|c|c|c|c|}
\hline Electrode & $R_{S}\left(\Omega \mathbf{c m}^{2}\right)$ & $R_{\mathrm{ct}}\left(\Omega \mathbf{c m}^{2}\right)$ & $Q\left(\mu F\right.$ cm $\left.^{2} s^{n-1}\right)$ & $\mathbf{n}$ \\
\hline Bare CPE & 6.84 & 207.90 & 35 & 0.77 \\
\hline $\mathrm{NXZ/CPE}$ & 6.01 & 168.66 & 28 & 0.73 \\
\hline MWCNT/CPE & 5.60 & 85.81 & 250 & 0.70 \\
\hline $\mathrm{AuNiSiO}_{2} / \mathrm{MWCNT} / \mathrm{CPE}$ & 5.52 & 53.96 & 120 & 0.68 \\
\hline AuNiNXZ/CPE & 5.21 & 40.60 & 14.7 & 0.79 \\
\hline NiNXZ/MWCNT/CPE & 5.20 & 34.65 & 82 & 0.78 \\
\hline AuNXZ/MWCNT/CPE & 5.10 & 32.31 & 1000 & 0.56 \\
\hline $\mathrm{AuNiNXZ}_{10} / \mathrm{MWCNT}_{25} / \mathrm{CPE}_{65}$ & 4.82 & 18.82 & 210 & 0.67 \\
\hline $\mathrm{AuNiNXZ}_{25} / \mathrm{MWCNT}_{10} / \mathrm{CPE}_{65}$ & 4.52 & 10.01 & 360 & 0.71 \\
\hline AuNiNXZ $25 / \mathrm{MWCNT}_{25} / \mathrm{CPE}_{50}$ & 4.40 & 4.97 & 79 & 0.91 \\
\hline
\end{tabular}

* CPE is given by $Z(\omega)=Q^{-1}(j \omega)^{-n}$ 


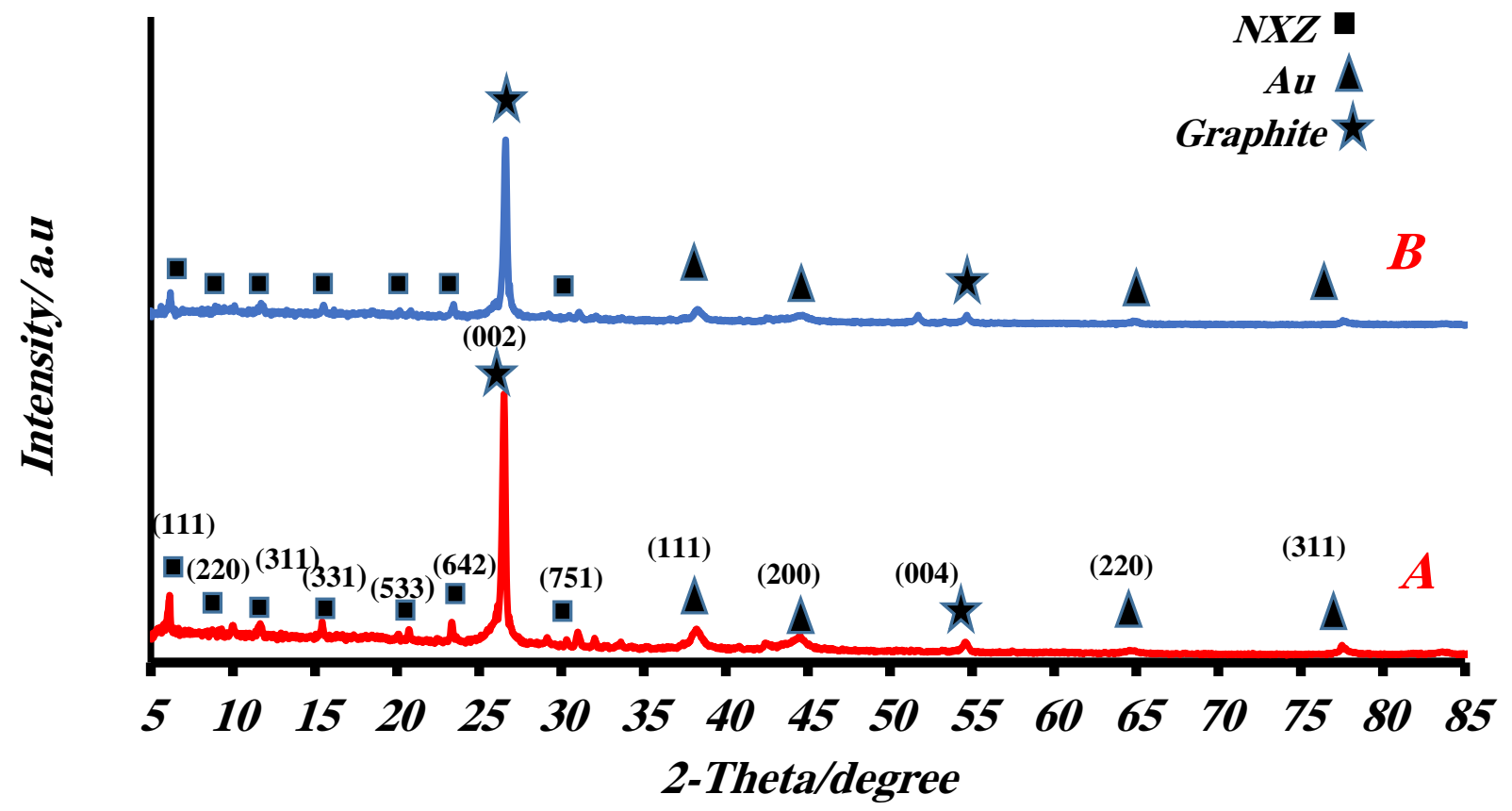

Fig. S5. XRD patterns of $A u N i N X Z_{25} / \mathrm{MWCNT}_{25} / \mathrm{CPE}_{50}$ before $(\mathrm{A})$ and after $(\mathrm{B})$ the chronoamperometric stability test. 

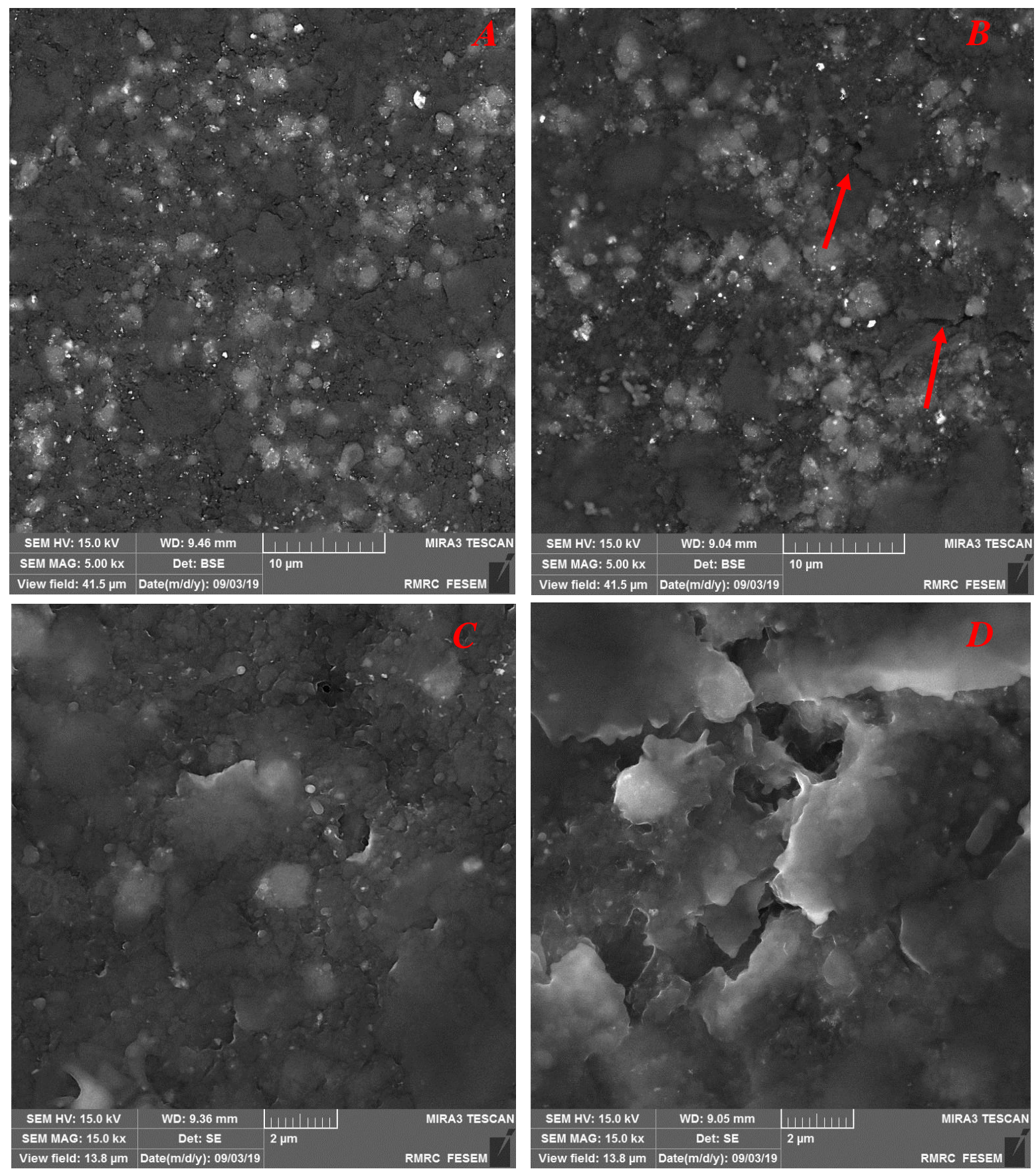

Fig. S6. FESEM images of AuNiNXZ $25 / \mathrm{MWCNT}_{25} / \mathrm{CPE}_{50}$ before (A and C) and after (B and D) the chronoamperometric stability test. 